\title{
Engineering and characterization of a symbiotic selection-marker-free vector-host system for therapeutic plasmid production
}

\author{
$\mathrm{XINCHANG} \mathrm{SHI}^{1}$ and JUNZHI WANG ${ }^{1,2}$ \\ ${ }^{1}$ Department of Biopharmaceutics, School of Pharmacy, Fourth Military Medical University, Xian, Shaanxi 710032; \\ ${ }^{2}$ National Institutes for Food and Drug Control, Beijing 100050, P.R. China
}

Received August 15, 2014; Accepted April 20, 2015

DOI: $10.3892 / \mathrm{mmr} .2015 .3945$

\begin{abstract}
The present study aimed to develop a symbiotic selection-marker-free plasmid and host system that would allow successful plasmid maintenance and amplification for use in gene therapy. Initially, the chromosomal aspartate-semialdehyde dehydrogenase (asd) gene was disrupted in DH10B Escherichia coli using Red recombinase-mediated homologous recombination. This method required the use of linear DNA fragments carrying kan-kil genes, and/or homologous extensions to the targeted locus. The resultant auxotrophic cell wall-deficient strain (DH10B $\Delta a s d)$ was evaluated as a symbiotic host for amplification of the marker-free plasmid, allowing it to supply ASD activity. In order to construct the plasmid, an asd expression cassette was inserted, under the control of the nirB promoter, into a eukaryotic expression vector, and its kanamycin resistance gene was subsequently removed. The symbiotic plasmid and host system was assessed for numerous plasmid production and stability parameters, including structure, yield, plasmid-retention rate, and bacterial storability, under various conditions. The presence of the plasmid was subsequently confirmed by growth test, restriction enzyme mapping, and sequencing. The plasmid yield and copy number produced in the symbiotic cells, in the absence of antibiotic selection, were shown to be similar to those produced under kanamycin selection, in the cells containing the precursor plasmid and kanamycin resistance gene. Furthermore, the results of the present study demonstrated that when inoculated with $<1 \%$ inoculant volume, $>98 \%$ of the cells in the culture retained the plasmid regardless of the number of passages. The strain was stable when stored at $-70^{\circ} \mathrm{C}$, with negligible viability loss over 12 months. The constructed plasmid is stable and
\end{abstract}

Correspondence to: Professor Junzhi Wang, National Institutes for Food and Drug Control, 2 Tiantan Xili, Beijing 100050, P.R. China E-mail: junzhiwang55@163.com

Key words: plasmid production, marker-free plasmid, homologous recombination, gene therapy, symbiotic plasmid-host system, plasmid retention has potential in future gene therapy, while much work is still required.

\section{Introduction}

Naked plasmid delivery systems have been recognized as an appealing alternative to viral vectors, due to their ability to avoid the immunogenicity and toxicity problems commonly associated with viral systems $(1,2)$. Since $1989,>1,800$ clinical trials have been approved worldwide, with $\sim 20 \%$ of these trials assessing the use of plasmid DNA as a vector system (3-5). DNA vectors generally encode antibiotic resistance genes in the plasmid backbone (6). Recently, the widespread use of antibiotics has resulted in the emergence of so-called super bacteria, which are resistant to multiple drugs (7). As a result of enhanced antibiotic management, the use of antibiotics has been banned in the production process of therapeutic plasmids in the European Union, except at the seeding stage. In addition, regulatory authorities in Europe and the US have voiced their concerns regarding the use of antibiotics or antibiotic selection markers in plasmids destined for clinical use $(8,9)$. Therefore, the development of various antibiotic marker-free selection approaches for the production of therapeutic plasmids has become an attractive solution (6).

Numerous strategies have been assessed regarding the production of antibiotic marker-free plasmids, including the use of auxotrophic complementation, toxin-antitoxin-based systems, RNA-based selection markers, and overexpression of growth essential genes (10). The present study aimed to develop a diaminopimelic acid (DAP) auxotrophy-based plasmid system, in order to produce plasmids for therapeutic use. In prokaryotes, the asd gene encodes aspartate-semialdehyde dehydrogenase (ASD; EC 1.2.1.11) (11). ASD is involved in catalyzing the intermediate steps leading to the synthesis of DAP, which in turn is an important component of the peptidoglycans that are used in cell wall synthesis by gram-negative bacteria. Bacteria that are deficient in ASD are unable to synthesize a functional cell wall, and therefore would be unable to grow, due to cell lysis $(12,13)$. Previous studies have reported the creation of auxotrophic S. typhimurium and E. coli strains with defects in the asd or QAPRTase genes for use in antibiotic marker-free plasmids $(14,15)$; however, their plasmid production-associated 
performance has not been fully characterized. Therefore, the present study aimed to engineer a widely used recombinant auxotrophic DH10B E. coli strain. The DH10B strain has previously been engineered for the propagation of large insert DNA library clones (16), and is commonly used throughout the research community due to its useful properties, including its high transformation efficiency, its ability to take up and stably maintain large plasmids, its lack of methylation-dependent restriction systems (MDRS), and its lacZ-based $\alpha$-complementation colony screening properties (17).

In the present study, the cell wall auxotrophic mutant DH10B $\Delta$ asd was generated to host a eukaryotic asd expression vector, thereby creating a balanced-lethal system similar to those reported previously $(18,19)$. Numerous parameters associated with the stability of the host, and the yield of the plasmid, were evaluated to determine the potential of the system in large-scale plasmid production.

\section{Materials and methods}

Media, chemicals and reagents. Ampicillin (Amp), streptomycin (Stm), spectinomycin (Spm), and kanamycin (Km)-resistant $\left({ }^{\mathrm{R}}\right)$ transformants were selected on a Luria Bertani (LB) agar medium (Kemin Bio Tech., Shanghai, China), containing the antibiotics at 100, 100,50, and $25 \mu \mathrm{g} / \mathrm{ml}$, respectively. All antibiotics were purchased from Sigma-Aldrich (St. Louis, MO, USA). DAP (Sigma-Aldrich) was used at $100 \mu \mathrm{g} / \mathrm{ml}$, and L-arabinose (Sigma-Aldrich) was used at $0.4 \%$. The oligonucleotides were purchased from Beijing Sunbiotech Co.,Ltd.(Beijing, China). The restriction enzymes were obtained from New England Biolabs Inc. (Ipswich, MA, USA), and the Taq Plus DNA polymerase was purchased from Takara Biotechnology Co., Ltd (Dalian, China).

Bacteria and plasmids. The DH10B (F-mcrA $\Delta(m r r-h s d \mathrm{RM}$ $\mathrm{S}-$ mcrBC) $\Phi 80$ lacZ $\mathrm{ZM} 15 \Delta$ lacX74 recAlendA1 araD139 $\Delta($ ara leu) 7,697 gal $\mathrm{U}$ galK rps $\mathrm{L}$ nup $\mathrm{G} \lambda$-) E. coli strain was purchased from Invitrogen Life Technologies (Carlsbad, CA, USA), and was maintained in LB medium. The pKD46 and pKan-kil plasmids were provided from Dr. Shanhu Li (China Academy of Military Medical Science), and were previously described (16), and the pDV-cmv-gene plasmid was constructed in our laboratory (unpublished data; China Institute for Food and Drug control).

Disruption of asd. A Red recombinase-based homologous recombination technique was used to knockout the chromosomal asd in the DH10B cells, as reported previously $(13,20)$. The details of the asd knockout are presented in Fig. 1. Briefly, transformants carrying a pKD46 Red helper plasmid were induced in $5 \mathrm{ml}$ super optimal broth (Hope Bio Tech., Qingdao, China) cultures with Amp and L-arabinose at $30^{\circ} \mathrm{C}$. The plasmid was subsequently transformed with linear DNA fragments carrying kan-kil and asd-homologous extensions using a Gene Pulser Xcell Electroporation system with $0.1 \mathrm{~cm}$ chambers according to the manufacturer's instructions (Bio-Rad Laboratories Inc., Hercules, CA, USA). The fragment was obtained by polymerase chain reaction (PCR), using Taq Plus DNA polymerases with the following primer sequences: Forward, 5'-ATTTATACAGCACACATCTTTGCAGGA AAAAAACGCTTATTAGTGAATGCTTTTGCTTG-3'; and reverse, 5'-CGC ACT AAC AGG GGC GGCATC GCG CCCCAGATTTAATGAACAACCTCCTTAGTACAT

GC-3', and pkan-kil as a template. The electroporated cells $\left(1 \times 10^{9}-2 \times 10^{9} \mathrm{CFU} / \mathrm{ml}\right)$ were plated out to select for the $\mathrm{Km}^{\mathrm{R}}$ and DAP-dependent transformants, and subsequently colony-purified at $37^{\circ} \mathrm{C}$, prior to being tested for Amp sensitivity to isolate the cells that had lost the helper plasmid. The helper plasmidcellswerethenmadecompetentagainandreceivedanother round of electroporation with the linear fragments carrying the homologous extensions without the kan-kil region. The fragment was obtained by PCR using Taq Plus DNA polymerases with the following primer (Sun Bio Tech, Beijing, China) sequences: Forward, 5'-CGGCACATTTATACAGCACACATCTTTGCA GGAAAAAAACGCTTTCATTAAATCTGGGG-3'; and reverse, 5'-CTGCGCTTACTCCTGTATTACGCACTAACA GGGGCGGCATCGCGCCCCAGATTTAATGA-3'. The PCR mixture was comprised of $12.5 \mu \mathrm{l}$ Q5 High-Fidelity 2X Master mix (Takara Biotechnology Co., Ltd), $10 \mu \mathrm{M}$ forward primer (1.25 $\mu \mathrm{l}), 10 \mu \mathrm{M}$ reverse primer $(1.25 \mu \mathrm{l}), 1 \mu \mathrm{l}$ template DNA and made up to $25 \mu \mathrm{l}$ with nuclease-free water. The transformants were subsequently induced at $37^{\circ} \mathrm{C}$ for $k i l$ expression and homologous recombination, in order to remove the kan-kil fragment that was previously integrated into the E. coli genome (Fig. 1).

$P C R$ verification. PCR was used to confirm that all mutants had the correct structure. A freshly isolated colony was suspended in $50 \mu \mathrm{l}$ water, from which $3 \mu \mathrm{l}$ aliquots were used in separate $25 \mu \mathrm{l}$ PCR reaction mixtures following a 2 min pre-incubation 'hot start' at $95^{\circ} \mathrm{C}$. The primers (Sun Bio Tech) used were as follows: Forward, 5'-ATTTATACAGCACACATCTTTGCAGGAAAA

AAACGCTTATTAGTGAATGCTTTTGCTTG-3'; and reverse, 5'-CGCACTAACAGGGGCGGCATCGCGCCCCAG ATTTAATGAACAACCTCCTTAGTACATGC-3'. Control colonies were tested side-by-side, and the amplified products were analyzed by agarose gel electrophoresis.

Construction of the antibiotic resistant-marker-free plasmid. Detailed steps for the construction of the plasmid are presented in Fig. 2. Briefly, the DNA sequence of the entire expression cassette, including the nirB15 promoter (21), the Shine-Dalgarno sequence, the asd coding region (Salmonella enterica subsp. enterica serovar typhi str. Ty2, GenBank: AAO71451.1), and the terminator was chemically synthesized to incorporate the appropriate restriction sites (Genewiz, Inc., South Plainfield, NJ, USA). The sequence was ligated into the pMD 18-T-simple plasmid (Sun Bio Tech) and confirmed by sequencing at the junction regions, prior to double digestion with KpnI and $B a m \mathrm{HI}$, in order to release the expression cassette. The released fragment was ligated to the similarly digested pDV-cmv-gene plasmid in order to generate pDV-cmv-gene-nirb-asd $\mathrm{Km}^{+}$. The $\mathrm{Km}$ resistance gene $\left(\mathrm{Km}^{+}\right)$in the resultant plasmid was then removed by digestion with HindIII and XhoI, and the purified plasmid was ligated by annealing a dsDNA fragment carrying complementary restriction sites to the linearized plasmid prior to ligation. The sequences of the dsDNA are as follows: Sense, 5'-TCGAGTAGATAACTGACTAGCATAACCCCT TGGGGCCTCTAAACGGGTCTTGAGGGGTTTTTTA-3'; and antisense, 5'-AGCTTAAAAAACCCCTCAAGACCC GTTTAGAGGCCCCAAGGGGTTATGCTAGTCAGTTATC 


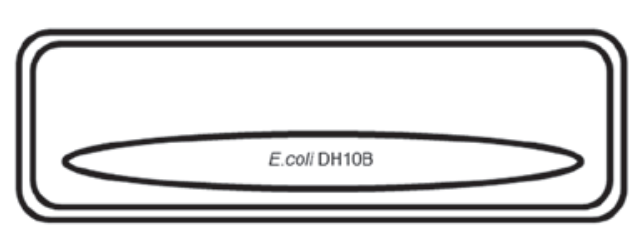

Amplified the kan/kil DNA fragment by PCR ( $95^{\circ} \mathrm{C}$ for $5 \mathrm{~min}$

30 cycles $\left[95 \mathrm{C}\right.$ for $30 \mathrm{~s}, 55^{\circ} \mathrm{C}$ for $30 \mathrm{~s}, 72 \mathrm{C}$ for $\left.1 \mathrm{~min}\right]$,

$30 \mathrm{~s}, 72 \mathrm{C}$ for $10 \mathrm{~min})$

with the primer for knocking out kan/kil

\begin{tabular}{|l|l|}
\hline Homologous arm & Homologous arm \\
\hline
\end{tabular}
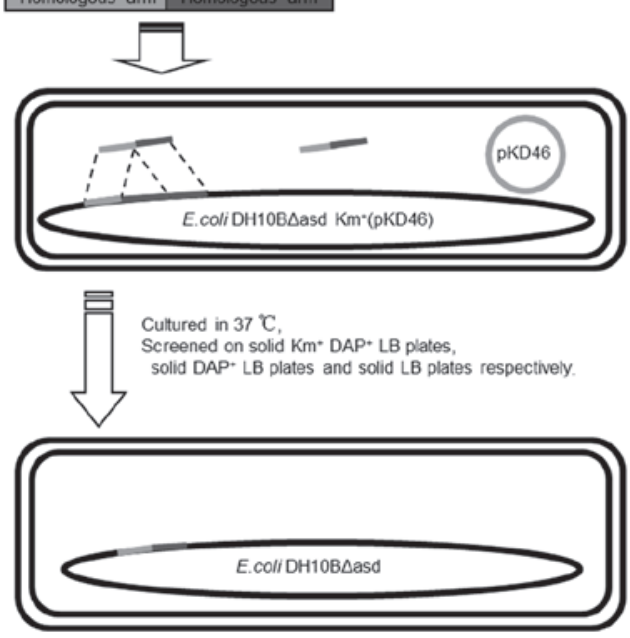

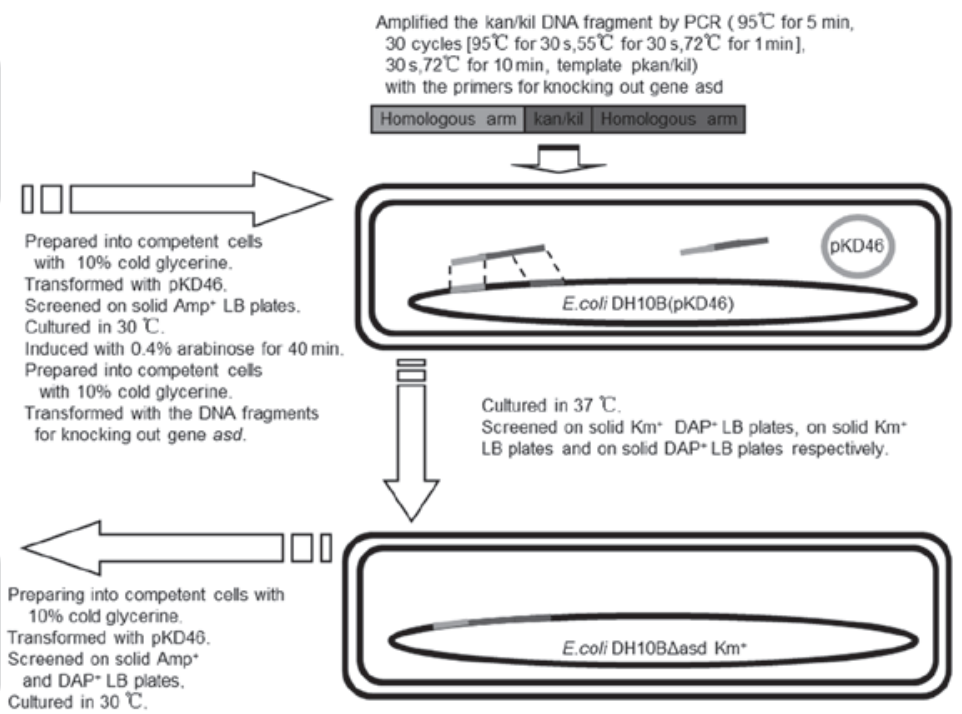

Induced with $0.4 \%$ arabinose for $40 \mathrm{~min}$.

Prepared into competent cells with

$10 \%$ cold glycerine.

Transformed with

the DNA fragment for knocking out kan/kil.

Figure 1. Schematic diagrams of the steps required to knock out the chromosomal asd gene in DH10B E. coli. PCR: Polymerase chain reaction; DAP, diaminopimelic acid; LB, Luria Bertani; Amp, Ampicillin; Km, Kanamycin.

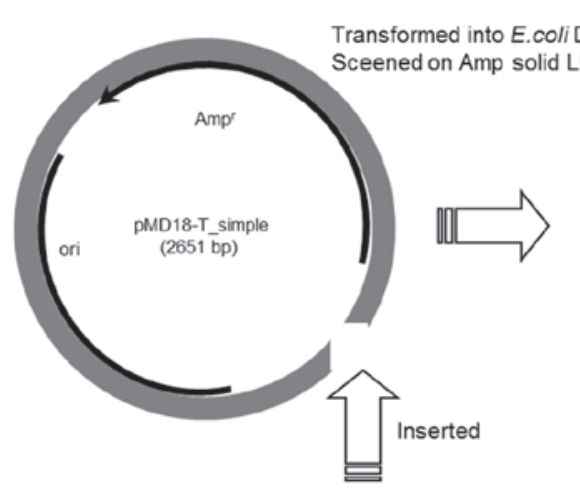

\begin{tabular}{|l|l|l|}
\hline NirB Promoter & asd & $\rho$ terminator \\
\hline
\end{tabular}

The dsDNA synthesized by PCR

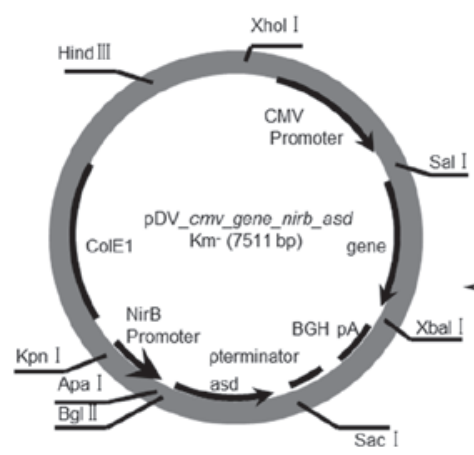

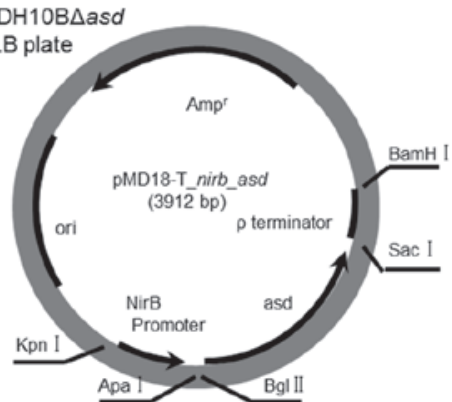

Digested by Kpn I and BamH I

$\checkmark$ Purified the DNA fragment including asd gene
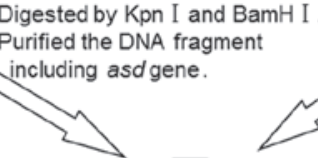

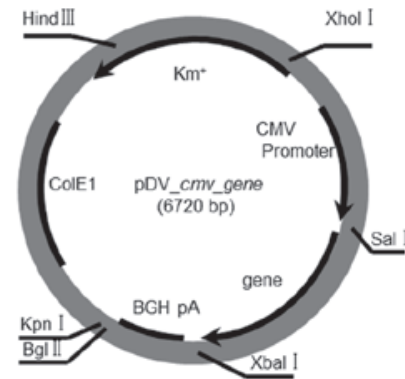

Digested by Kpn I and Bgl II Purified the linear vector fragment

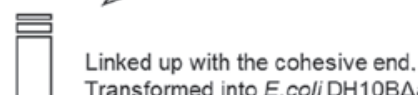

Transformed into E.coliDH10BAasd. Sceened on Km,DAP solid LB plate.

Digested by Hind III and Xhol I inked up with the dsDNA of the primers annealing. Sceened on DAP solid LB plate.
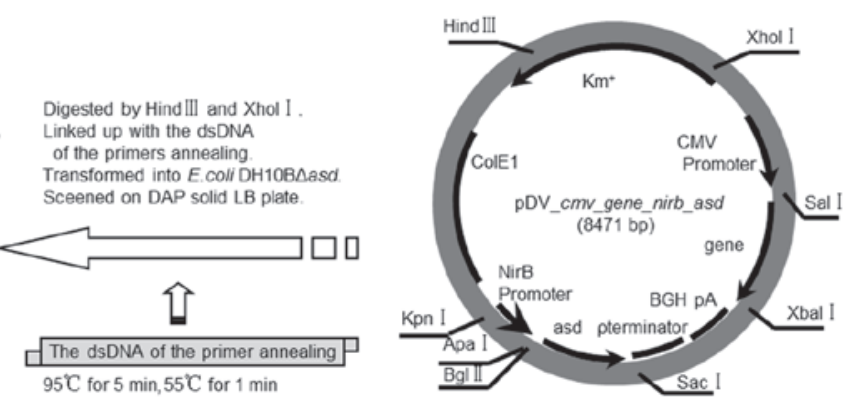

Figure 2. Schematic diagrams of the method used to construct the antibiotic-resistant-marker-free vector pDV-cmv-gene-nirb-asd Km- DAP, Diaminopimelic acid; LB, Luria Bertani; Amp, Ampicillin; Km, Kanamycin. 


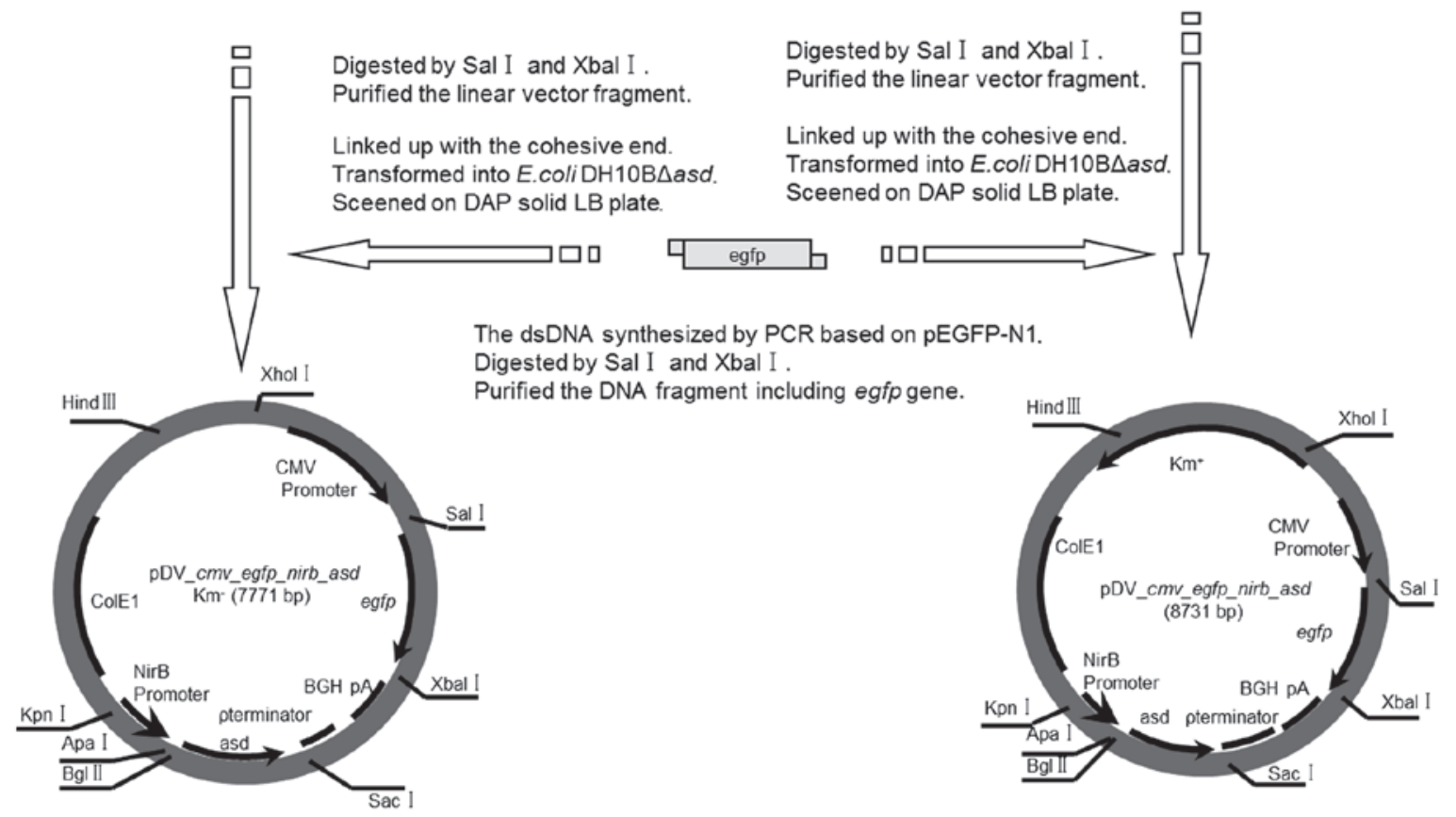

Figure 3. PCR, polymerase chain reaction; DAP, diaminopimelic acid; LB, Luria Bertani; EGFP, enhanced green fluorescent protein.

TAC-3'. The ligation products were subsequently transformed into the DH10B $\triangle$ asd cells and spread onto LB agar plates to recover DAP-independent colonies, prior to being further colony tested on LB agar plates for Km-sensitivity, in order to identify any potential antibiotic resistant-marker-free vectors.

Cell growth test. DH10B $\triangle$ asd cells were streaked onto agar plates containing $\mathrm{LB}, \mathrm{DAP}^{+} \mathrm{LB}, \mathrm{Stm}^{+} \mathrm{DAP}{ }^{+} \mathrm{LB}$, and $\mathrm{Spm}^{+} \mathrm{DAP} \mathrm{P}^{+} \mathrm{LB}$, and their growth was subsequently recorded after overnight culture at $37^{\circ} \mathrm{C}$.

Cell lysis test. To test the autolysis of DH10B $\Delta$ asd cells the cells were grown on $\mathrm{DAP}^{+} \mathrm{LB}$ agar plates, prior to being washed three times with sterile distilled water, and were then resuspended in sterile distilled water. Three types of media containing: Water only, LB, and LB + Amp, were inoculated with $100 \mu \mathrm{l}$ aliquots prior to culture at $37^{\circ} \mathrm{C}$. Subsequently, $10 \mu \mathrm{l}$ aliquots were taken from each culture at various times, and plated onto LB agar medium, prior to overnight incubation at $37^{\circ} \mathrm{C}$. For each assessment three colony plates were counted to determine the number of colonies.

Plasmid structure and sequence analysis. To verify the structure of the expected plasmid, purified pDV-cmv-gene-nirb-asd $\mathrm{Km}^{-}$DNA was digested in three double enzyme reactions: SalI $+B g l \mathrm{II}, H i n \mathrm{dIII}+B g l \mathrm{II}$, and HindIII + SalI. The digested products were subsequently analyzed by agarose gel electrophoresis. The selected clones were fully sequenced on both strands at the Beijing Genomics Institute (Beijing, China).

Plasmid yield and supercoiled plasmid. The DH10B $\Delta$ asd cells containing pDV-cmv-gene-nirb-asd $\mathrm{Km}^{+}$and pDV-cmv-gene-nirb-asd $\mathrm{Km}^{-}$were cultured overnight at $37^{\circ} \mathrm{C}$ with agitation at $150 \mathrm{rpm}$, in liquid LB medium with or without $\mathrm{Km}(100 \mu \mathrm{g} / \mathrm{ml})$. The cells were then harvested by centrifugation and diluted with $0.9 \% \mathrm{NaCl}$ solution with the same $\mathrm{OD}_{600}$ values for both cultures $(\mathrm{OD}=2.0)$. Plasmid DNA was prepared from $2 \mathrm{ml}$ aliquots of the cells $\left(1 \times 10^{9}-2 \times 10^{9} \mathrm{CFU} / \mathrm{ml}\right)$ using the Qiagen Mini-prep kit (Qiagen, Inc., Valencia, CA, USA) and quantified using a UV spectrometer (DU800; Beckman Coulter, Inc., Fullerton, CA, USA) at a wavelength of $260 \mathrm{~nm}$. The plasmids were subsequently separated on $1 \%$ agarose gel, and the resulting images were scanned and analyzed to determine the amount of DNA in the supercoiled conformation using MultiGauge (Version 3.1, Fujifilm, Tokyo, Japan).

Plasmid retention. After 10, 20 and 30 passages, cells from the rapidly growing cultures were taken and inoculated in LB medium with inoculant-medium ratios of 1:1,000, 1:100 and 1:10. The cells were subsequently cultured for $5 \mathrm{~h}$ at $37^{\circ} \mathrm{C}$ with $150 \mathrm{rpm}$ agitation. $\mathrm{LB}$ agar and $\mathrm{DAP} \mathrm{P}^{+} \mathrm{LB}$ agar plates were then spread with $100 \mu 1$ aliquots from each of the cultures prior to further overnight culture at $37^{\circ} \mathrm{C}$. The number of colonies on both plates were counted and used to calculate the percentage of the bacterial cells that had retained the plasmid and could grow normally in LB medium.

Doubling time. Aliquots of $10 \mu \mathrm{l}$ of cells were removed following $0,1.5,3$ and $4.5 \mathrm{~h}$ cultures in LB broth with $200 \mathrm{rpm}$ agitation at $37^{\circ} \mathrm{C}$. The cells were subsequently diluted 100 times, spread onto $\mathrm{LB}$ agar plates, and cultured at $37^{\circ} \mathrm{C}$. Following overnight growth, the colonies were counted and used to calculate the cell doubling time using the Gompert function $\left(\mathrm{F}=\mathrm{K} \cdot \mathrm{a}^{\mathrm{b} \cdot \mathrm{t}}\right)(22)$.

Storages. DH10B $\Delta$ (asd $\mathrm{pDV}$-cmv-gene-nirb-asd $\mathrm{km}^{-}$) cells were stored as glycerol stock at $25,4,-20$ and $-70^{\circ} \mathrm{C}$ for 12 months and their survival rate was subsequently tested by plating $100 \mu \mathrm{l}$ of the thawed cells onto LB agar plates, prior to counting the number of colonies formed after overnight culture. 


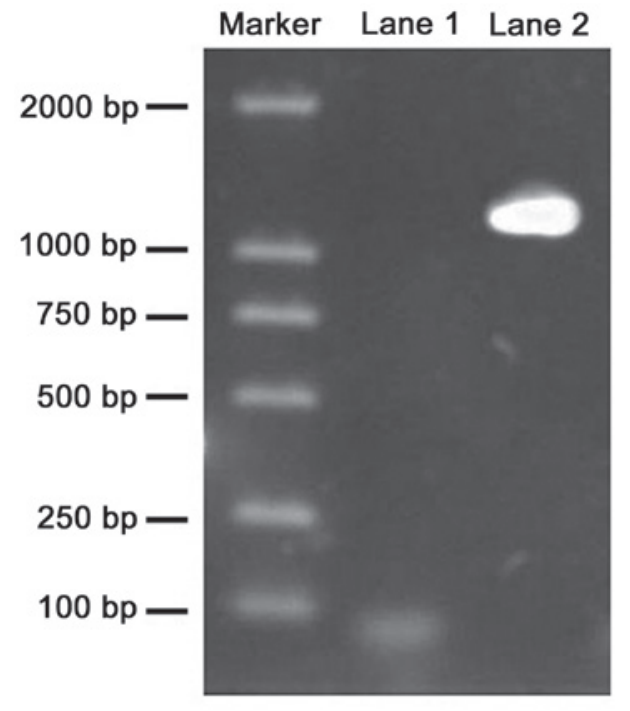

Figure 4. Agarose gel electrophoresis of the polymerase chain reaction amplification products for the asd gene in E. coli cells. Lane 1, asd deletion candidate; Lane 2, DH10B wildtype cells.

Construction of egfp expression antibiotic resistant-marker-free plasmid and lipofection of HeLa cells. The egfp gene was ligated into the pDV-cmv-gene-nirb-asd $\mathrm{Km}^{-}$and pDV-cmv-gene-nirb-asdKm-plasmids (Fig. 3), and the resulting transformants containing the egfp gene were selected by PCR, and sequenced using egfp gene-specific primers (Sun Bio Tech). Based on the concentration and molecular mass, the copies of the two types of plasmid were adjusted to the same order of magnitude. The lipofection of the HeLa cells with the two plasmids was carried out using Lipofectamine ${ }^{\circledR}$ reagent (Invitrogen Life Technologies), according to the manufacturer's instructions. $\mathrm{ddH}_{2} \mathrm{O}$ was used as a control. The egfp gene was also ligated into the pDV-cmv-gene-nirb-asd and pDV-cmv-gene plasmids, which were then transformed into the DH10BA $\Delta$ asd E. coli strain. DH10B $\triangle$ asd cells were streaked onto agar plates containing LB medium, and their growth was recorded after overnight culture at $37^{\circ} \mathrm{C}$.

Statistical analysis. The data were analyzed using SPSS version 19.0 (IBM SPSS, Armonk, NY, USA), and P<0.05 was considered to indicate a statistically significant difference. Curve fitting was conducted using OriginPro v7.0 (OriginPro, Northampton, MA, USA).

\section{Results}

Screening for asd disruption mutants. Following several preliminary gene disruption experiments, namely the use of the pKD46 plasmid to provide Red recombinase and FRT-flanked $\mathrm{Km}$ resistance gene fragments carrying the homologous extensions to asd, hundreds of colonies were obtained that could grow on LB medium supplemented with Km and DAP. Thus indicating that the $\mathrm{Km}$ gene was successfully inserted into the DH10B genome. The Km-resistant cells were subjected to a second round of recombination by electroporating a linear DNA fragment containing asd-homologous extensions without the kan-kil region, in order to remove the kan-kil fragment

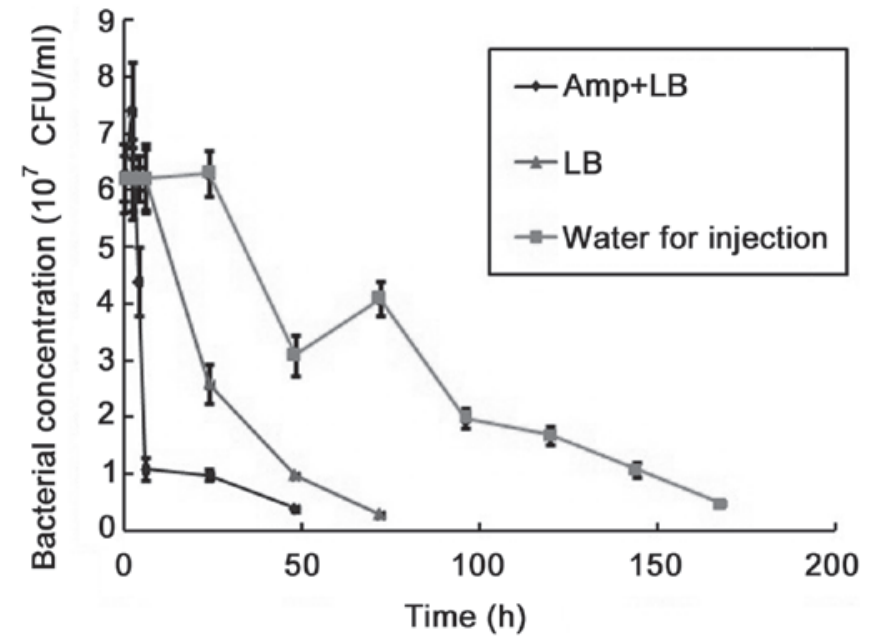

Figure 5. Survival rates measured as colony formation units of E. coli $\mathrm{DH} 10 \mathrm{~B} \Delta$ asd in various media. Amp, ampicillin; LB, Luria Bertani.

that had previously been inserted into the bacterial genome. Following several experiments used to comparatively screen the transformants on LB agar plates containing $\mathrm{Km}^{+} \mathrm{DAP}^{+}$, $\mathrm{DAP}^{+}$, and LB only, a few colonies were obtained that could grow on $\mathrm{DAP}^{+} \mathrm{LB}$, but not on $\mathrm{Km}^{+} \mathrm{DAP}^{+} \mathrm{LB}$, or $\mathrm{LB}$. These results indicate that the cells in these colonies had lost resistance to $\mathrm{Km}$ as a result of the secondary recombination, and were dependent on the exogenous supply of DAP. This is due to the expected disruption of asd in the strain. The colonies were therefore selected as candidates for $a s d$-deficient lines for further confirmation at the DNA level.

PCR verification. asd locus-specific PCRs were performed using genomic DNA extracted from several candidate template colonies. As shown in Fig. 4, a band corresponding to the expected 77 bp product was observed in the asd deletion candidate colony, whereas a 1,184 bp PCR product was amplified with DNA from the wildtype DH10B cells, indicating that asd-specific disruption had occurred in the cell line. Therefore, the cell line was named DH10B $\Delta a s d$.

Cell lysis test. Deletion or disruption of functional asd in E. coli cells is expected to result in the failure of the bacterium to synthesize a functional cell wall, due to the disruption of DAP synthesis, thus resulting in cell death. To acquire a better understanding regarding how cell death occurred in the asd-disrupted cells, cell survival duration was determined in various growth conditions by exposing them to water and LB media, with or without Amp, for various durations. As demonstrated in Fig. 5, the cells gradually lost their viability following exposure to these media, but at different rates. The half-life of the cells was $\sim 2-3 \mathrm{~h}$ and $\leq 1$ day in the LB medium with and without Amp, respectively. In distilled water, the half-life of the cells was 3-4 days.

Construction of marker-free expression vectors. The $\mathrm{pDV} c m v$ gene was used as a starter plasmid for construction of an antibiotic resistance-marker-free vector. Following several steps of DNA manipulation, as described in the Materials and Methods, the DH10B $\Delta$ asd cell colonies that contained the 
Table I. Plasmid retention ratios of the asd deletion mutant strain after various generations of passages, and after culture at various dilutions.

\begin{tabular}{lccr}
\hline & \multicolumn{3}{c}{ Dilution at inoculation } \\
\cline { 2 - 4 } Passage & $1: 1,000$ & $1: 100$ & $0.82 \pm 0.04$ \\
\hline 10 & $1.00 \pm 0.01$ & $0.98 \pm 0.02$ & $0.83 \pm 0.03$ \\
20 & $0.99 \pm 0.02$ & $0.97 \pm 0.03$ & $0.80 \pm 0.05$ \\
30 & $0.99 \pm 0.01$ & $0.98 \pm 0.03$ & 0.03 \\
\hline
\end{tabular}

Ratios were calculated as (number of colonies appeared on LB medium)/(number of colonies appeared in LB + DAP medium), and expressed as the mean \pm standard deviation from three independent experiments.

Table II. Colony forming ability of the DH10B $\Delta$ asd (pDV-cmv-gene-nirb-asd $\mathrm{km}^{-}$) cells at various storage temperatures, and after various durations.

\begin{tabular}{lcccc} 
& \multicolumn{4}{c}{ Colony forming units/ml culture after storage at certain temperatures } \\
\cline { 2 - 5 } $\begin{array}{l}\text { Storage } \\
\text { months })\end{array}$ & $-70^{\circ} \mathrm{C}$ & $-20^{\circ} \mathrm{C}$ & $4{ }^{\circ} \mathrm{C}$ & $25^{\circ} \mathrm{C}$ \\
\hline 0 & $6.4 \times 10^{8} \pm 0.5 \times 10^{8}$ & $6.4 \times 10^{8} \pm 0.5 \times 10^{8}$ & $6.4 \times 10^{8} \pm 0.5 \times 10^{8}$ & $6.4 \times 10^{8} \pm 0.5 \times 10^{8}$ \\
1 & $6.5 \times 10^{8} \pm 0.5 \times 10^{8}$ & $5.1 \times 10^{8} \pm 0.4 \times 10^{8}$ & $1.6 \times 10^{8} \pm 0.2 \times 10^{8}$ & $2.1 \times 10^{4} \pm 0.2 \times 10^{4}$ \\
3 & $6.4 \times 10^{8} \pm 0.4 \times 10^{8}$ & $2.5 \times 10^{8} \pm 0.4 \times 10^{8}$ & $1.1 \times 10^{7} \pm 0.4 \times 10^{7}$ & $5.0 \pm 2.0$ \\
6 & $6.2 \times 10^{8} \pm 0.3 \times 10^{8}$ & $7.9 \times 10^{7} \pm 0.3 \times 10^{8}$ & $1.6 \times 10^{5} \pm 0.2 \times 10^{5}$ & - \\
12 & $6.0 \times 10^{8} \pm 0.5 \times 10^{8}$ & $1.4 \times 10^{7} \pm 0.2 \times 10^{7}$ & $50.0 \pm 4.0$ & - \\
\hline
\end{tabular}

Results are expressed as mean \pm standard deviation from three independent experiments.

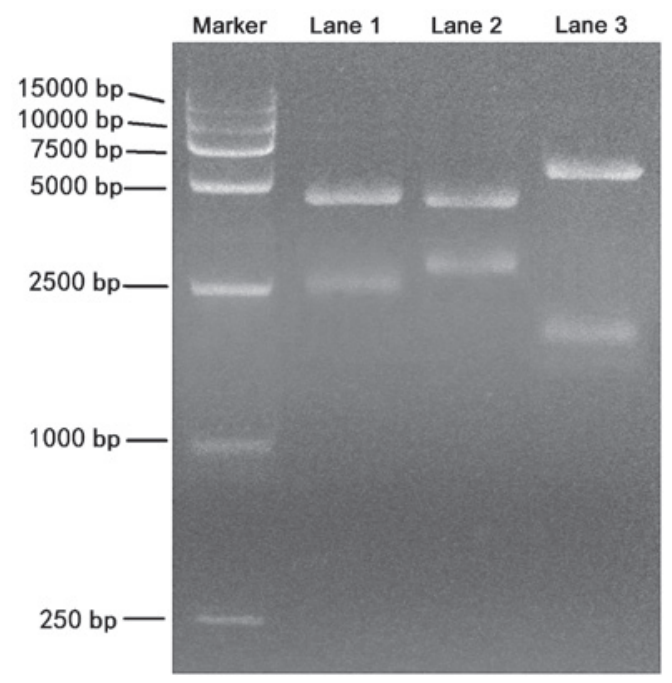

Figure 6. Agarose gel analysis of the restricted pDV-cmv-gene-nirb-asd $\mathrm{Km}$ plasmid. Lane 1, SalI + BglII (2675 bp + 4583 bp); Lane 2, BglII and HindIII (2928 bp + 458 bp); Lane 3, HindIII + SalI (1908 bp + 5603 bp).

target plasmid pDV-cmv-gene-nirb-asd $\mathrm{Km}^{-}$were recovered, since they could grow in the LB medium without requiring extra supplementation of DAP. The nirB-asd cassette was expected to provide ASD activity in the DH10B $\Delta$ asd cells. To verify the structure of the plasmid, DNA was prepared from a number of colonies and was restricted using restriction enzymes in three reactions: SalI + BglII, HindIII + BglII, and HindIII + SalI . The digested products were analyzed and plasmids with the expected bands and sizes were identified (Fig. 6). Sequencing of the plasmids further confirmed that the entire sequences were correct (data not shown).

Plasmid yield and conformation. To investigate whether the elimination of antibiotic pressure during the plasmid amplification process would have an impact on the plasmid yields in this symbiotic system, the plasmid yield of the resultant constructs were compared prior to and following the removal of $\mathrm{Km}^{+}$in the backbone, in LB medium with or without $\mathrm{Km}$ in the DH10B $\Delta$ asd cells. The results demonstrated that the plasmid yields were similar in the two culture conditions (0.527, vs. $0.552 \mu \mathrm{g} / \mathrm{ml}$ of culture in LB with or without $\mathrm{Km}$, respectively), and did not statistically significantly differ from each other $(\mathrm{P}>0.05)$. The examination of the conformation of the plasmids extracted from the cultures under the two conditions demonstrated a similar percentage $(\sim 60 \%)$ of supercoiled plasmid DNA (data not shown).

Plasmid retention. Differing from the typical plasmid amplification protocols, where antibiotics are added to the culture media in order to selectively proliferate the bacterial cells that contain plasmids bearing antibiotic resistant genes, maintenance of the plasmids in this symbiotic system relies solely on DAP production from the host plasmid containing an asd 

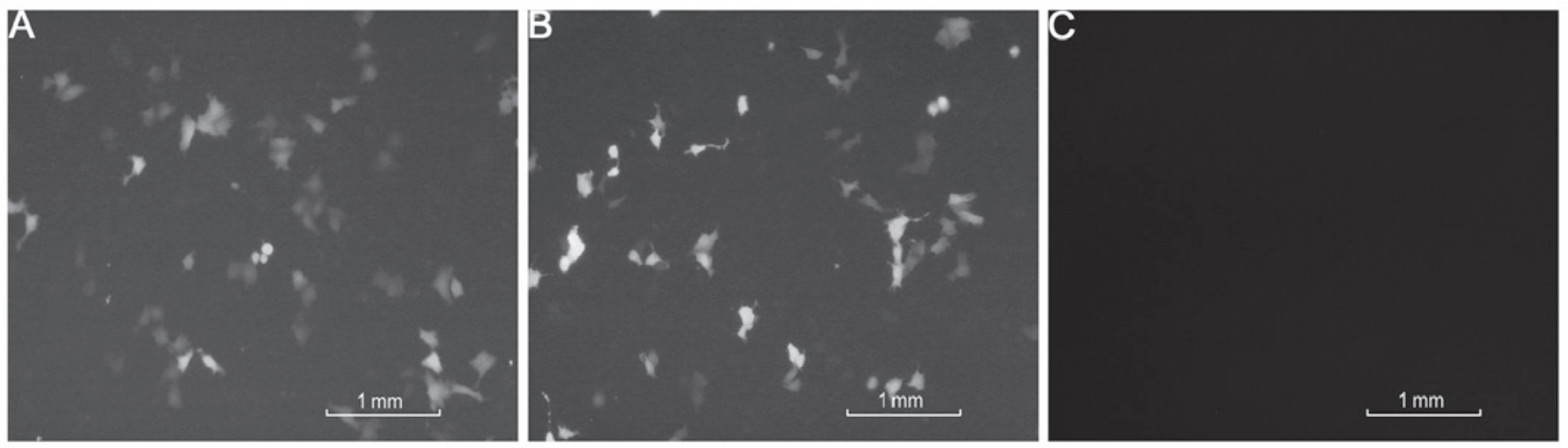

Figure 7. Expression of plasmid pDV-cmv-egfp-nirb-asd $\mathrm{Km}^{-}$and pDV-cmv-egfp-nirb-asd in HeLa cervical cancer cells. HeLa cells with green fluorescence after transfection with (A) pDV-cmv-egfp-nirb-asd Km-plasmid and (B) plasmid pDV-cmv-egfp-nirb-asd, and (C) without green fluorescent protein expression after transfected with physiological saline.

expression cassette located in the asd-disrupted cells. It is therefore important to investigate how well the plasmid can be retained under the symbiotic, but non-selective condition. The cells containing $\mathrm{pDV}-\mathrm{cm}$-gene-nirb-asd $\mathrm{Km}^{-}$were grown to various passages in LB medium, and were then plated out onto LB agar plates with or without DAP after diluting the culture up to 1,000 times. The colonies that appeared in LB medium were considered to contain the plasmid, whereas those that appeared on the LB + DAP medium were considered to be either with or without plasmid. The results shown in Table I indicate that the plasmid retention rates (ratio of colony counts on medium without and with DAP) were statistically the same (100, vs. $99 \%)$ between the 10th and the 30th passages when they were inoculated at a 1:1,000 dilution. However, the plasmid retention rates were reduced to $\sim 80 \%$ when inoculated at a 1:10 dilution.

Bacterial storability. As a cell line deficient in cell wall composition, it is important to analyze bacterial storability at various temperatures for its further use in a plasmid production system. The results summarized in Table II demonstrated that the cells rapidly lost their viability when stored at higher temperatures $\left(25^{\circ}\right.$ and $\left.4^{\circ} \mathrm{C}\right)$, but maintained stable colony forming ability when stored at $-70^{\circ} \mathrm{C}$. The half-life of the cells was 2 and 15 days when the cells were stored at $25^{\circ} \mathrm{C}$ and $4^{\circ} \mathrm{C}$, respectively, and increased to 2 months when stored at $-20^{\circ} \mathrm{C}$, and to 10 years when stored at $-70^{\circ} \mathrm{C}$.

Doubling time. To examine the growth speed that would impact overall plasmid productivity, the present study determined the number of cells in the culture by counting their colony forming units at various time points. The doubling time computed based on the compertz function, was 29.9 min when the cells were grown in LB medium.

Expression of egfp gene in HeLa cells. The expression of enhanced green fluorescence protein (EGFP) is shown in Fig. 7. The cells transfected with the pDV-cmv-egfp-nirb-asd $\mathrm{Km}$ plasmid exhibited green fluorescence under blue light, indicating a good expression of EGFP protein in the cells. As assessed with the naked eye, the efficiency of transfection with the two types of plasmid appeared similar. The DH10B $\Delta$ asd cells with the pDV-cmv-egfp-nirb-asd gene grew well on LB medium, whereas the cells transformed with the pDV-cmv-egfp gene did not, which is indicative of a normally functioning asd gene following the insertion of egfp.

\section{Discussion}

Due to increasing concerns regarding horizontal antibiotic resistance gene transfer, it has become crucial to develop plasmid vectors that are free of antibiotic resistance genes for potential use in human gene therapy $(6,7,10)$. The present study aimed to develop an antibiotic resistant-marker-free vector that can be produced in large quantities, in a similar manner to regular plasmids that carry antibiotic selection makers. This can be achieved so that the system has both a specific host that can retain and amplify the plasmid, and a plasmid that can provide all of the essential elements for survival of the host cells. Such systems have previously been explored in S. typhimurium and E coli $(6,10,18,20)$. In the present study, using two rounds of Red recombinase-induced homologous recombination, the asd gene was initially knocked out in the $E$. coli cells, in order to render them dependent on exogenous DAP supply. A plasmid was subsequently constructed that contained an asd expression cassette to provide ASD once transformed into the cells. Therefore, a symbiotic plasmid production system that met all of the targets was generated.

Initially, an asd-disrupted strain was engineered from commonly used DH10B E. coli cells. As a laboratory strain for prokaryotic plasmid amplification, the strain has a ribosomal mutation conferring resistance to Stm, which is useful for selective bacterial maintenance and many other routine features, including large scale plasmid production $(6,16,17)$. It also avoids any potential issues associated with JM83 plasmid instability, the derivative of which was used to construct the asd-deficient strain in the early study (14). In order to disrupt endogenous asd expression in the DH10B cells, a modified prophage recombination system was used to introduce a Km-resistance gene into the DH10B cells, as previously reported $(13,20)$. The successful functioning of this step was demonstrated by the large number of $\mathrm{Km}$ resistant colonies that were recovered following the electroporation of linear DNA fragments containing the kan-kil genes. The kan-kil fragment 
contains a DNA sequence consisting of an aminoglycoside phosphotransferase expression cassette for $\mathrm{Km}$ resistance (23), and a $\lambda$ phage lysis gene cassette under the control of a heat-inducible promoter (24). The former was used to select homologous recombination events targeting the asd gene, and the latter was used to remove the introduced Km-resistance gene by killing the non-recombinants in the second round of homologous recombination. However, removal of the inserted $\mathrm{Km}$ resistance gene appeared to be poorly effective, and most of the colonies recovered at the beginning of the study were false positives. These results were likely due to the low efficiency of kil gene lysis, due to the low copy or mostly single copy number of the gene within the cells. To overcome this, several rounds of induction at $37^{\circ} \mathrm{C}$ followed by the plating of the transformants onto LB medium supplemented with DAP was subsequently performed. Following several attempts, a small number of colonies were eventually obtained and their presence confirmed by asd locus-specific PCR and positive $\mathrm{Km}$ sensitivity tests. Exposure of the cells to water and LB medium with and without Amp led to cell death, indicating that the loss of asd had compromised cell survival. These results were most likely due to the inability of the cells to synthesize a functional cell wall. However, as opposed to antibiotic-induced cell death, the loss of viability in these cells appeared to be slow, particularly when cultured in water. These observations were likely due to the residual DAP in the cells, and the low metabolic activity of the cells in water, which prevented them from actively synthesizing a new cell wall. Nevertheless, further investigation is required in order to elucidate the effects of asd disruption on cell wall synthesis.

The nirB promoter was chosen to provide ASD activity and to drive asd expression, as it has previously been demonstrated to exhibit leaking expression in aerobic conditions (25), thus allowing many of the screenings and tests to be carried out under normal aerobic conditions. The starter plasmid pDV-cmv gene contained a relax ColE1 replicon $(26,27)$, and would therefore provide between 300 and 500 copies of the nirB promoter to direct the expression of the cassette in the $E$. coli cells. This resulted in the leaked production of ASD at a sufficient enough quantity to allow DAP to be synthesized for cell wall integrity. Therefore, under aerobic conditions, DH10B $\Delta$ asd cells transformed with the marker-free plasmid $\mathrm{pDV}$-cmv-gene-nirb-asd $\mathrm{Km}^{-}$were expected to grow normally, even in the absence of DAP. The experiments demonstrated that colonies appeared on the LB medium in the absence of DAP whilst containing the correct form of pDV-cmv-gene-nirb-asd $\mathrm{Km}^{-}$(Fig. 6), thus indicating successful symbiosis in the vector/host combination. Yield and conformation of the plasmid DNA was also found to be similar when produced in the symbiotic condition under antibiotic selection, indicating that symbiosis is robust enough to replace antibiotic selection for plasmid production. Furthermore, plasmids containing the asd gene were constructed with EGFP expression, to test whether the plasmids would function regularly in other cells. In the present study HeLa cells were chosen for this experiment. The results indicated that the plasmid was expressed normally in the HeLa cells, and that the insertion of the egfp gene into the plasmid did not influence asd gene function.

One particularly important consideration for an antibiotic resistant-marker-free plasmid production system is how well the plasmid can be maintained in the host cell in the absence of negative selection from antibiotics, whilst relying solely on symbiosis. The results of the present study demonstrated that the plasmid can be retained in the asd-disrupt cells without noticeable loss for $\leq 30$ generations of cell divisions when inoculated at a 1:1,000 (v-v, inoculants: Medium) ratio. However, the plasmid loss became evident when the inoculation ratio was 1:10 (Table I). This may be due to cross-cell feeding of DAP from the cells with the plasmid to the cells that had lost the plasmid, thereby increasing the chance of survival of the cells that had lost the plasmid. Such cross-feeding would be dependent on the number of cells within the plasmid (such as the cells in the inoculants), leading to an increase in plasmid loss in an inoculant quantity-dependent manner as seen in the present study and as reported previously (27).

Another feature that would have a marked impact on the practical use of this system is the survivability and storability of the asd-deficient cells, particularly in the case of long term storage. While these cells quickly lost their viability at room or refrigerator temperature $\left(25\right.$ and $\left.4^{\circ} \mathrm{C}\right)$, little change was observed in viability after 12 months storage at $-70^{\circ} \mathrm{C}$ (Table 2), indicating that they may be stored for long term use. This also implies that the disruption of asd would not compromise the viability of the cells, as long as the lost asd function is complemented by the plasmid. The pDV-cmv-gene-nirb-asd $\mathrm{Km}^{-}$plasmid is expected to be of a value similar to that of the eukaryotic expression vector pVXA1, which can be used in preventive research such as the production of melanoma and Alzheimer's DNA vaccines, as well as in the treatment of hemagioma and artery terminal ischemia using animal expression models.

In conclusion, the present study developed a symbiotic vector/host system for the use of marker-free plasmids in gene therapy. The vector may be further manipulated in order to carry additional gene(s) of interest for various purposes, and may be produced in large quantities in the symbiotic host cells under antibiotic-free conditions. This method would considerably reduce the risk of antibiotic resistant gene transfer during the therapeutic process, and would markedly improve therapeutic safety.

\section{Acknowledgements}

The present study was financially supported by the National Health and Family Planning Commission of the People's Republic of China (grant no. 2012ZX09304010), and the National High-tech R\&D Program, Ministry of Science and Technology of the People's Republic of China (grant no. 2012AA020805).

\section{References}

1. Tolmachov OE: Building mosaics of therapeutic plasmid gene vectors. Current gene therapy 11: 466-478, 2011.

2. Nahaei M, Valizadeh H, Baradaran B, et al: Preparation and characterization of chitosan/beta-cyclodextrin nanoparticles containing plasmid DNA encoding interleukin-12. Drug research (Stuttg) 63: 7-12, 2013.

3. Przybylowski M, Bartido S, Borquez-Ojeda O, Sadelain M and Riviere I: Production of clinical-grade plasmid DNA for human Phase I clinical trials and large animal clinical studies. Vaccine 25: 5013-5024, 2007 
4. Norell H, Poschke I, Charo J, et al.: Vaccination with a plasmid DNA encoding HER-2/neu together with low doses of GM-CSF and IL-2 in patients with metastatic breast carcinoma: a pilot clinical trial. J Transl Med 8: 53, 2010.

5. Smith LR, Wloch MK, Ye M, et al.: Phase 1 clinical trials of the safety and immunogenicity of adjuvanted plasmid DNA vaccines encoding influenza A virus H5 hemagglutinin. Vaccine 28 2565-2572, 2010.

6. Oliveira PH and Mairhofer J: Marker-free plasmids for biotechnological applications - implications and perspectives. Trends Biotechnol 31: 539-547, 2013.

7. Pruden A, Pei R, Storteboom H and Carlson KH: Antibiotic resistance genes as emerging contaminants: Studies in northern Colorado. Environ Sci Technol 40: 7445-7450, 2006.

8. 7.0 EP: Gene transfer medical products for human use. 648,2011

9. U.S. Food and Drug Administration: Guidance for human somatic cell therapy and gene therapy. 1998.

10. Vandermeulen G, Marie C, Scherman D and Préat V: New generation of plasmid backbones devoid of antibiotic resistance marker for gene therapy trials. Mol Ther 19: 1942-1949, 2011

11. Haziza C, Stragier P and Patte JC: Nucleotide sequence of the asd gene of Escherichia coli: Absence of a typical attenuation signal. EMBO J 1: 379-384, 1982.

12. Narayanan K and Warburton PE: DNA modification and functional delivery into human cells using Escherichia coli DH10B Nucleic Acids Res 31: e51, 2003.

13. Datsenko KA and Wanner BL: One-step inactivation of chromosomal genes in Escherichia coli $\mathrm{K}-12$ using PCR products. Proc Natl Acad Sci USA 97: 6640-6645, 2000.

14. Curtiss R III, Galan JE, Nakayama K and Kelly SM Stabilization of recombinant avirulent vaccine strains in vivo. Res Microbiol 141: 797-805, 1990.

15. Dong WR, Xiang LX and Shao JZ: Novel antibiotic-free plasmid selection system based on complementation of host auxotrophy in the NAD de novo synthesis pathway. Appl Environ Microbiol 76: 2295-2303, 2010.

16. Durfee T, Nelson R, Baldwin S, et al: The complete genome sequence of Escherichia coli DH10B: Insights into the biology of a laboratory workhorse. J Bacteriol 190: 2597-2606, 2008.
17. GrantSG,Jessee J,Bloom FR and Hanahan D: Differential plasmid rescue from transgenic mouse DNAs into Escherichia coli methylation-restriction mutants. Proc Natl Acad Sci USA 87: 4645-4649, 1990.

18. Galán JE, Nakayama K and Curtiss R III: Cloning and characterization of the asd gene of Salmonella typhimurium: Use in stable maintenance of recombinant plasmids in Salmonella vaccine strains. Gene 94: 29-35, 1990.

19. Zhao Z, Wang C, Xue Y, et al: Immunogenicity and protective efficacy of pertactin recombinants against Bordetella bronchiseptica challenge. Wei Sheng Wu Xue Bao 50: 1239-1245, 2010 (In Chinese)

20. Arias-Lopez C, Lazaro-Trueba I, Kerr P, et al: p53 modulates homologous recombination by transcriptional regulation of the RAD51 gene. EMBO Rep 7: 219-224, 2006.

21. Makoff AJ and Oxer MD: High level heterologous expression in E. coli using mutant forms of the lac promoter. Nucleic Acids Res 19: 2417-2421, 1991.

22. Teeter KC, Thibodeau LM, Gompert Z, Buerkle CA, Nachman MW and Tucker PK: The variable genomic architecture of isolation between hybridizing species of house mice. Evolution 64: 472-485, 2010.

23. Kobayashi F, Yamaguchi M and Mitsuhashi S: Drug resistance to aminoglycosidic antibiotics in Pseudomonas aeruginosa and its lability. Jpn J Microbiol 16: 425-431, 1972.

24. Yagisawa $M$, Yamamoto $H$, Naganawa $H$, Kondo $S$ and Takeuchi T: A new enzyme in Escherichia coli carrying R-factor phosphorylating 3'-hydroxyl of butirosin A, kanamycin, neamine and ribostamycin. J Antibiot (Tokyo) 25: 748-750, 1972.

25. Wang H and Gunsalus RP: The nrfA and nirB nitrite reductase operons in Escherichia coli are expressed differently in response to nitrate than to nitrite. J Bacteriol 182: 5813-5822, 2000.

26. Bowers LM, Lapoint K, Anthony L, Pluciennik A and Filutowicz M: Bacterial expression system with tightly regulated gene expression and plasmid copy number. Gene 340: 11-18, 2004.

27. Wu YC and Liu ST: A sequence that affects the copy number and stability of pSW200 and ColE1. J Bacteriol 192: 3654-3660, 2010. 